\title{
Introductory psychology laboratories using graphic simulations of virtual subjects
}

\author{
HERBERT A. COLLE \\ Wright State University, Dayton, Ohio \\ and \\ RANDALL F. GREEN \\ Stargate, Dayton, Ohio
}

\begin{abstract}
Graphical simulations of the behavior of virtual subjects in laboratory or field microworlds were developed to teach research principles to introductory psychology students. Students conduct studies in laboratory classes, assuming the primary role of being an experimenter. Microworld modules were designed to foster the flexible exploration of important steps in the research process, including hypothesis generation, research planning and design, behavioral observation or testing, and data summarization and analysis. The modules were designed to impart a sense of behavioral "presence" so that students would obtain an appreciation of realistic behavioral observation and of testing methodologies. Microworlds that have been developed include infant preferential looking (cognitive processes), courting behavior of fireflies, personality test development, working memory, operant conditioning, singlecell recording of sensory function, and testing of brain-damaged people. Principles that guided the development of software modules and laboratory class exercises are discussed.
\end{abstract}

At most American universities, the first course in introductory or general psychology is taught in large lecture classes. This is a cost-effective method for conveying a large amount of information, but didactic lectures are less effective at conveying procedural knowledge about how psychological research is conducted. In other sciences, laboratory sections are used for this purpose. Laboratory exercises have been used in introductory or general psychology courses (Goolkasian, 1988; Kornfield, 1989), but they are still uncommon, apparently being used more often at primarily undergraduate teaching institutions (Hornby \& Anderson, 1994).

The present paper describes an approach that was developed at Wright State University to provide a large number of students with laboratory experience. Its goal was to enhance the students' understanding of the process of conducting psychological research. Introductory psychology at Wright State University is a general education course that is required of all students, not just of psychology majors or students with related majors, and most students enroll in the course during their first year. Currently, over 2,000 students per year complete introductory psychology laboratories in an administrative format similar to that used by other lower level science courses; large lecture classes of 400 introductory psychology students are accompanied by 2 -contact-hour laboratory sessions $(3$

Correspondence concerning this article should be addressed to $\mathrm{H}$. A. Colle, Department of Psychology, Wright State University, Dayton, $\mathrm{OH}$ 45435 (e-mail: hcolle@nova.wright.edu).

Note: The authors may have a direct financial interest in some of the software described in this paper.-Editor. credit-hours of lecture and 1 credit-hour of laboratory). Each laboratory session has 32 students and is led by a graduate teaching assistant in classrooms that contain 16 computers, each one being used by a pair of students.

\section{General Course Goals}

Several very broad goals have guided the development of the laboratories. First, laboratory exercises should represent the major topic areas of introductory psychology. Experiments in cognition and perception are heavily used in many laboratory courses in psychology, especially computer-based ones, because they are easy to implement in classroom laboratories with undergraduate students. However, courses in introductory psychology cover other topics, such as personality, animal behavior, and developmental psychology. Laboratories should include these topics; otherwise, students will obtain only a limited perspective of the research techniques and methodologies that are used by psychologists.

Second, laboratories should focus on having students use the logic, procedures, and methods of scientific psychologists. Students should obtain procedural knowledge by generating hypotheses, setting up conditions to test them, obtaining relevant data or observations that are reliable and not biased, and then interpreting the data after they have been collected. However, a technical understanding of equipment, specialized calibration techniques, complex experimental designs, and statistics are not needed for understanding and appreciating the scientific process, and it may misdirect attention from important aspects of the procedures.

Third, laboratories should require that students act as researchers, focusing their attention on what a psycholo- 
gist would do to conduct a study or to test a hypothesis, not primarily on experiencing psychological phenomena. For example, they should develop and evaluate personality tests, not take them as subjects. Students spend much of their time in many laboratory classes acting as subjects in order to generate data, but this role is very different from the role of experimenter. An experimenter focus does not rule out having an experiential component in laboratories, but it should not be the dominant feature if you want to teach scientific thinking.

Finally, laboratories should provide realistic research experiences, with students getting a sense of the activities of observation and testing. Participating in research activities is important, not just verbally describing methodological steps that can be conveyed in lectures or textbooks and evaluated in exams. Classroom laboratory activities should mirror research laboratory activities as much as possible.

\section{Laboratory Microworlds}

The above goals dictated the use of simulations in introductory psychology laboratories because there are cost, coordination, technical, or ethical problems with conducting many laboratory or field studies and with using large numbers of babies, patients, or rodents for classroom purposes. On the other hand, software simulations that existed did not satisfy the above four goals. Therefore, we developed "virtual" research environments with software programs to meet these goals. Each software module covered research on a different topic area or conceptual domain, and it provided students with an opportunity to perform the major steps of a research project in that conceptual domain. The modules that were developed do not control a student's course of action, as instructional programs do. Instead, each module creates a microworld arena in which students operate as experimenters, one designed so that they can navigate flexibly back and forth through its segments.

Flexibility was built into the overall structure of the programs. Scientific research activity was divided into four steps or subgoals: hypothesis generation, research planning and design, data collection, and data analysis/ interpretation. Labeling and visually separating these steps of the scientific method should help students to focus on these as subgoals in solving microworld research problems and, therefore, to be more likely to use them when faced with new research problems (Catrambone, 1994). Each of the four major research steps was made accessible via a different function key. Hypothesis generation activities occur in the HYP screen, accessed with F2. Research planning and setup activities occur in the PLAN screen, accessed with F3. Observation and data collection activities occur in the RUN screen, accessed with F4. Data summary, analysis, and graphing activities occur in the DATA screen, accessed with F5. The F1 function key is used to store and print data. Pressing a function key immediately moves the student-experimenter into that screen and activity. Therefore, students can move freely back and forth between these activities to complete their research project. The software also was designed so that students were free to move about within each of these screens and perform on-screen activities flexibly.

\section{RUN Screen}

The RUN screen defines a conceptual domain from the point of view of the student. It determines what the student-experimenter observes and the data that can be collected. RUN screens should give students a clear sense of what it feels like to collect the data in a real research laboratory. This issue is critical for successful implementation of the graphic displays in microworld simulations, and it is discussed more completely below.

Infant observation. The RUN screen in each module was designed to capture behavior in a limited domain of a topic area. For example, Figure 1 shows the RUN screen from the infant observation laboratory in which studentexperimenters test 5-month-old simulated infants and observe their looking behaviors in order to evaluate their perceptual and cognitive abilities. A laboratory manual provides background information and an overview of the testing situation, including a diagram that shows that the infant is seated facing a stimulus display. A camera, directed at the infant's face from behind the display, captures the infant's head and eye movements. Images from the camera are displayed on a monitor in the control room; the RUN screen in Figure 1 shows this monitor, which student-experimenters use to observe the infant, usually to determine when the infant is looking at the stimulus display.

Figure 1 shows a static image; however, during the microworld simulation, the monitor in the RUN screen presents a behaving infant. From time to time, the infant's head turns left and right as well as up and down to various extents in a quasi-random fashion, controlled by a program that simulates the dynamics of how these motions occur in human infants. Likewise, eye movement dynamics were simulated using quasi-random left-right

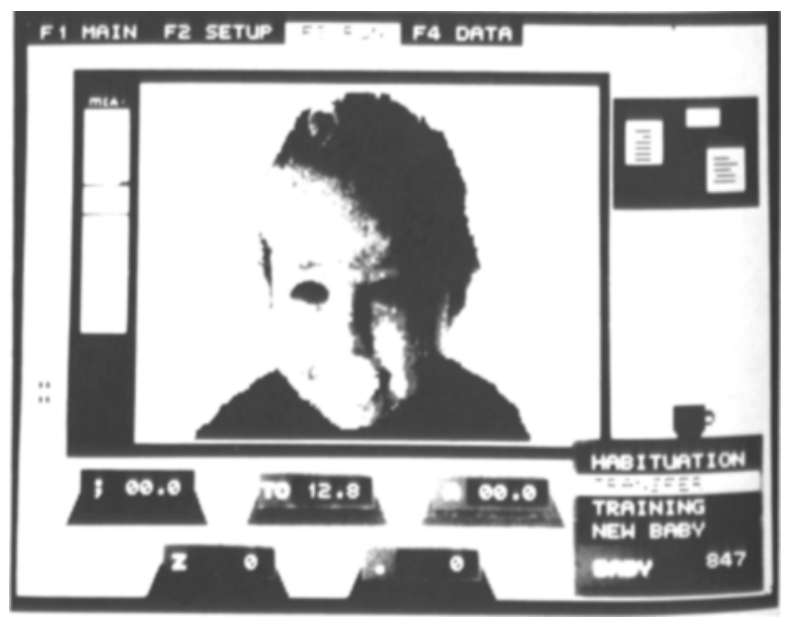

Figure 1. The RUN screen in the infant observation microworld. The infant's looking behavior is observed on the monitor in the control room. 
and up-down movements as well as realistic blink rates. Purkinje images are represented on the eyeballs because they are important for judgments of eye position. Infants also suck a pacifier and make gross body movements, such as rocking back and forth.

Depending upon stimulus conditions, a simulated infant will be more or less likely to attend to the stimulus object. Student-experimenters use the clocks and counters at the bottom of the screen as tools to measure and record these looking behaviors. Interobserver reliability can be estimated by comparing the times obtained from two simultaneous student-observers who have independent control over separate clocks. Students also can compare their observations with those of a simulated trained observer (TO). Of course, other behavior patterns in the microworld, such as sucking rate, blinking rate, or head movements, can be observed and recorded.

Student-experimenters use the experimenter's console on the bottom right-hand side of the RUN screen shown in Figure 1 to present a trial either using the habituation stimulus or using the transfer stimulus. Habituation and transfer stimuli are specified in the PLAN screen (F3). The experimenter's console also allows the current subject to be replaced with a new experimentally naive subject. Thus, simple perceptual preference tests may be conducted using either between-subject or within-subject designs. In addition, more complex transfer designs may be used to determine the stimuli to which habituation will generalize; by using appropriate procedures, infant cognitive capabilities, such as the recognition of object permanence, may be evaluated (Spelke, 1988).

Other microworld domains. Similar design principles were used to develop other microworld simulations. A firefly microworld introduces the student to field observations, as well as to laboratory research. Students study flash exchanges that occur during firefly courting behavior that precedes mating. They may observe the flash patterns and other activity of fireflies in field observations, or they may bring fireflies into the laboratory to systematically evaluate the characteristics of firefly behavior during the flash exchanges.

An operant conditioning and rat behavior microworld allows students to observe rat behavior in a small cage and to explore operant conditioning methods. Simulated rats perform characteristic rodent behaviors, such as walking, sniffing, rearing, face washing, scratching, and eating. Students use a tool kit of counters, clocks, and a notepad, as well as a cumulative recorder to record rat behavior. They may condition rats to perform simple activities, including pressing a lever, by delivering rewards using the method of successive approximations (shaping), or they may explore how rats behave under different schedules of reinforcement or experimental extinction. Our operant conditioning microworld, although it was developed in parallel over 4 years ago, is similar to the simulation recently described by Graham, Alloway, and Krames (1994).

A short-term memory (serial recall) microworld allows both an experimental and an experiential component simultaneously. One student in a pair may act as an experimenter, testing a simulated subject and recording his/her behavior. At the same time, the RUN screen may include a second test station so that the other student may be tested as a second subject. Responses of simulated subjects approximate the error patterns, such as serial position curves and reversals, that are expected of human subjects. Simulated subjects may be tested in large group sessions as another option. Group testing is also used by the personality test development microworld. A personality trait structural model with a quasi-random probability response generator is used to simulate how subjects endorse test items.

\section{Model Development}

Microworld simulations are controlled by a model for the behavior being simulated and by using experimental conditions to control parameters of the model. In the infant observation microworld, a probabilistic finite state model was developed. The state space included the locations of head, eyes, and stimulus objects. An attentional model was overlaid so that state probabilities would depend upon the simulated infant's attentional state, which, in turn, depended upon the experimental manipulations and the displays.

A conceptual model is sufficient if it generates behavior that is expected under the experimental conditions being tested. It need not be theoretically correct or parsimonious. Model development is simplified because the set of parameters used to test the model is fixed in the microworld. Conditions that could falsify the model do not have to be included. Therefore, if a body of research knowledge exists, a microworld may be developed to simulate the results. Simulations may require complex rules, such as those described by Gonzalez (1994). However, the rules, once developed, can be coded in computer programs readily.

Conceptual state models must be converted into behavior that is dynamically displayed on the screen. Available RAM space determined the total number of images that could be utilized, because an entire microworld program, including all function key screens and all graphic and sound images, is stored and executed from RAM. As a result, display movements were smooth and the microworlds were responsive to student actions, without the delays that would have been imposed by disk or CD-ROM access. In our microworlds, most graphic screens have been broken into image components that are directly controlled and sequenced by image control drivers so as to save space and to obtain better image control. Thus, eye movements are controlled by eye image drivers. Blending and transitioning are controlled at this level in order to obtain smooth-looking image movements. Images were deliberately simplified and graphic compression schemes were specially designed in order to greatly reduce the amount of memory needed to store images. Although only $1 \mathrm{Mb}$ of RAM was available on the 80286 systems for which these microworlds were developed, infant behavior that appeared realistic could be simulated by using all of these techniques. 


\section{Fidelity}

The infant observation microworld was described above in some detail to provide a flavor for the approach. Because RUN screens should give students a clear sense of what it feels like to collect the data in a real research laboratory, it would be ideal if virtual reality systems could be used for teaching (see Durlach \& Mavor, 1995). Virtual environments attempt to create a subjective experience of "presence," which occurs when operators feel like they are actually present in the virtual world, not at an artificial interface. In a virtual laboratory environment, students should get a clear sense that they are in a laboratory or field environment observing subjects. However, presence in this strong sense may be too strict as a requirement. Simulations may be very effective without being completely virtual. Several dimensions appear to be important, including autonomy, interaction, and presence, and they can be described as varying continuously across artificial environments (Zeltzer, 1992). In simulator training, a similar concern has been addressed as simulator fidelity. A simulation has high fidelity if it closely resembles and acts like the world it is simulating. However, fidelity may be described on several different dimensions, and the minimum level of fidelity needed for effective training is a complex question (Hays \& Singer, 1989).

The issues of fidelity and presence are important for the development of microworld laboratories. Although microworld simulations run on relatively primitive 80286 computers, it has been our experience that students obtain a real sense of the dynamics of the behavior being studied. Presence is too strong to describe this feeling. Instead, students appear to find the simulated subjects "engaging" or creatures for which they could develop "empathy."

Two factors appear to be important in getting students to react to simulated subjects as if they were real: sufficient but not necessarily very high graphic fidelity, and sufficient behavioral fidelity. Simply stated, babies should look like babies and babies should act like babies. Perceptually, the image may be grainy as long as the infant's overall appearance is natural. Behavior fidelity is probably even more important. Simulations should include probabilistically determined actions, and the action set should be rich enough with significant actions so that subjects do not appear to be one dimensional. For example, Psychworld's operant conditioning simulation using a stick-figure pigeon with a very limited behavioral repertoire has very little perceptual and behavioral fidelity, and it does not appear to be effective for teaching science (Leith, Hamm, Duncanson, \& Senical, 1988).

\section{PLAN Screen}

The PLAN screen is used for research planning and setup activities. Selections made here determine the test conditions utilized in the RUN screen. For example, several different sets of stimulus objects may be tested in the infant preferential looking microworld. Checkerboard patterns may be used to assess the importance of perceptual complexity. In contrast, a rabbit that moves back and forth, going behind solid or notched screens, may be used to assess object permanence, a cognitive competency. Of course, the characteristics of attentive looking are different in these two cases. The specific task stimuli to be used during habituation or transfer and test conditions, such as trial duration or experimenter-blind testing, also are selected.

The PLAN screens in the other microworlds are comparable. In operant conditioning and rat behavior, the PLAN screen determines which schedule of reinforcement will be used. It also can be used to select a rat with previous experience (e.g., previously shaped rats), the deprivation level (percent body weight), and the time of day. By manipulating time of day, the diurnal cycle of rat activity may be studied. In the firefly microworld, variables such as calendar date, time of day, and geographic location may be selected for field studies, while variables such as species, gender, and wavelength of the test light may be manipulated in laboratory studies. In the short-term memory microworld, list properties such as modality can be controlled. The PLAN screen for the personality test development microworld is a workspace for creating, editing, and storing previously created tests. These personality tests are evaluated for test-retest reliability or are intercorrelated with each other using the RUN screen.

The PLAN screen is also used to provide students with expert advice about observations that require critical judgment. For example, videotapes of firefly field observations of male-female interactions may be selected for viewing. In these videotapes, the location of the female firefly is clearly marked, because female fireflies are difficult to find in a normally busy field. Similarly, a student may watch a simulated expert shape the behavior of a rat at different stages of the shaping procedure, in order to understand when a rat should be rewarded at a given stage. Judgments of when an infant is attending to a stimulus are not obvious; a simulated trained observer can provide his or her judgment to the students. In all modules, the use of expert advice is optional.

\section{HYP Screen}

The HYP screen is used to propose hypotheses relevant to the microworld's cognitive domain. As with the other screens, the student performs activities via menu selections. Student-experimenters create a hypothesis by selecting an independent variable from the Independent Variable menu, by selecting a dependent variable from the Dependent Variable menu, and by selecting a hypothesized relationship between them from the Relationship menu. A verbal statement of the hypothesis is displayed in the HYPOTHESIS display above these three menus, together with a schematic drawing of the function with the axes labeled correctly. HYP screens help students learn how to formulate hypotheses and encourage their formation. Ideally, this behavior would generalize, and students would formulate hypotheses whenever they face a scientific problem.

\section{DATA Screen}

The DATA screens are used for data summary, analysis, and graphing activities. The data collected in RUN 
screens are stored in data sets as records that can be scrolled through. A Command menu allows descriptive statistics, such as means, medians, standard deviations, and correlation coefficients, to be displayed and immediately presented as $x-y$ graphs of two variables. Introductory psychology students have used these tools with little difficulty.

\section{Instructional Activities}

A major advantage of microworlds is that students, including younger junior high school students who have participated in summer precollege programs, have found them to be inherently interesting. They appear to enjoy observing and interacting with virtual subjects. Microworlds provide an environment that encourages active participation and exploration (Papert, 1987; Shute \& Glaser, 1990). Interactions with virtual subjects also introduce videogame-like characteristics that are intrinsically motivating (Driskell \& Dwyer, 1984; Goble, Colle, \& Holland, 1988; Lepper, 1985).

Our introductory psychology course emphasizes understanding the basics of methodology. The seven microworlds have been used together with three other nonmicroworld laboratories: spatial rotation, forgetting, and Piagetian testing. A written laboratory manual guides students by presenting background information and an orientation to the modules. Exercises are provided along with examples of data tables and graphs. As with any instructional tool, effectiveness is determined by how the tool is used. To date, our evaluations have only been formative and design-oriented; students use and comment on program operation, or specific program components are evaluated. Student ratings of laboratory sections have been very high.

The same microworlds, however, could be used differently in introductory courses for honors students or for psychology majors or in advanced courses such as experimental psychology or research methods courses. In these cases, an instructor might want to spend several weeks on some of the microworlds and have students more thoroughly explore methodological issues or have students get the feel for pursuing a research problem with a bigger scope by using a series of related experiments. As an instructional tool, the microworld approach leaves these educational decisions to course instructors, not to software designers. As computers get more powerful, microworlds should be increasingly used at all levels of the undergraduate curriculum in psychology.

\section{Availability}

The simulation software described above is expected to be available in June 1996, for all IBM-compatible machines with $8028612 \mathrm{MHz}$ or higher CPUs. Plans are to distribute it inexpensively. To be put on a mailing list to receive information when it is available, write to $\mathrm{H}$. A. Colle, 1907 Southlawn Drive, Fairborn, OH 45324.

\section{REFERENCES}

Catrambone, R. (1994). Improving examples to improve transfer to novel problems. Memory \& Cognition, 22, 606-615.

DRISKELL, J. E., \& DWYER, D. J. (1984). Microcomputer videogame based training. Educational Technology, 24, 11-16.

DURLACH, N. I., \& MAVor, A. S. (EDS.) (1995). Virtual reality: Scientific and technological challenges. Washington DC: National Academy Press.

Goble, L. N., Colle, H. A., \& Holland, V. M. (1988). Development and evaluation of a computerized hand-held instructional prototype (CHIP). In Proceedings of the Sixth Conference on Interactive Instructional Delivery (pp. 859-864). Warrenton, VA: Society for Applied Learning Technology.

GonZALEZ, F. A. (1994). Simulations of schedule-controlled behavior. Behavior Research Methods, Instruments, \& Computers, 26, 224-230.

Goolkasian, P. (1988). A computerized laboratory for general psychology. Teaching of Psychology, 15, 98-100.

Graham, J., Alloway, T., \& Krames, L. (1994). Sniffy, the virtual rat: Simulated operant conditioning. Behavior Research Methods, Instruments, \& Computers, 26, 134-141.

HAYS, R. T., \& SINGER, M. J. (1989). Simulation fidelity in training system design. New York: Springer-Verlag.

HoRNBY, P. A., \& ANDERSON, M. D. (1994). Computer use in psychology instruction: A survey of individual and institutional characteristics. Behavior Research Methods, Instruments, \& Computers, 26, 250-254.

Kornfield, A. D. (1989). A review and student evaluation of Psychology on a Disk. MicroPsych Network, 4, 3-5.

Leith, C. R., Hamm, H. D., Duncanson, C., \& Senical, T. (1988). A computer-driven undergraduate laboratory curriculum and related support logistics. Behavior Research Methods, Instruments, \& Computers, 20, 188-190.

LEPPER, M. R. (1985). Microcomputers in education: Motivational and social issues. American Psychologist, 40, 1-18.

PAPERT, S. (1987). Microworlds: Transforming education. In R. W. Lawler \& M. Yazdini (Eds.), Artificial intelligence: Vol. 1. Learning environments and tutoring systems (pp. 79-94). Norwood, NJ: Ablex.

SHUTE, V., \& GlaSER, R. (1990). A large-scale evaluation of an intelligent discovery world: Smithtown. Interactive Learning Environments, 1, 51-77.

SPELKE, E. S. (1988). Where perceiving ends and thinking begins: The apprehension of objects in infancy. In A. Yonus (Ed.), The Minnesota symposia on child psychology (pp. 197-234). Hillsdale, NJ: Erlbaum.

ZelTZER, D. (1992). Autonomy, interaction, and presence. Presence: Teleoperators \& Virtual Environments, 1, 127-132.

(Manuscript received November 13, 1995; revision accepted for publication February 16, 1996.) 